Published in final edited form as:

J Am Geriatr Soc. 2016 February ; 64(2): 323-329. doi:10.1111/jgs.13948.

\title{
Emergency care utilization and the Medicare hospice benefit for patients with poor-prognosis cancer
}

\author{
Ziad Obermeyer, MD, MPhil ${ }^{1,2,3}$, Alissa C. Clarke, AB $^{2}$, Maggie Makar, BS ${ }^{2}$, Jeremiah D. \\ Schuur, MD, MHS ${ }^{1,2}$, and David M. Cutler, $\mathrm{PhD}^{4}$ \\ ${ }^{1}$ Department of Emergency Medicine, Harvard Medical School, Boston, MA \\ ${ }^{2}$ Department of Emergency Medicine, Brigham \& Women's Hospital, Boston, MA \\ ${ }^{3}$ Ariadne Labs, Brigham and Women's Hospital and Harvard School of Public Health, Boston, MA \\ ${ }^{4}$ Department of Economics, Harvard University, Cambridge, MA, and National Bureau of \\ Economic Research, Cambridge, MA
}

\begin{abstract}
Background/Objective-There is increasing interest in the relationship between palliative and emergency care. We compared patterns of Emergency Department (ED) utilization and inpatient admission rates for elderly patients with poor-prognosis cancers who enrolled in hospice to those of similar patients who did not.
\end{abstract}

Design-Matched case-control study.

Setting-Nationally-representative sample of Medicare fee-for-service beneficiaries with poorprognosis cancers who died in 2011.

Participants-Hospice beneficiaries, matched to non-hospice beneficiaries on time from poorprognosis cancer diagnosis to death; region; age; and sex.

Measurements-ED utilization and inpatient admission rates before and after hospice enrollment for hospice beneficiaries, compared to equivalent control periods.

Results-Of 272,832 matched beneficiaries, $81 \%$ visited the ED in the last 6 months of life. At baseline, daily ED utilization and admission rates were not significantly different between hospice and non-hospice beneficiaries. By the week before death, non-hospice patients averaged 69.6 ED visits/1000 beneficiary-days vs 7.6 for hospice beneficiaries (rate ratio: 9.7, 95\% CI: 9.3-10.0). Inpatient admission rates in the last week of life were $63 \%$ for non-hospice and $42 \%$ for hospice beneficiaries (rate ratio: $1.51,95 \%$ CI: $1.45-1.57$ ). Of all hospice beneficiaries, $28 \%$ enrolled

Corresponding author: Ziad Obermeyer, MD, MPhil, Department of Emergency Medicine, Harvard Medical School, Neville House, 75 Francis St, Boston, MA 02115 phone: 617-525-3133 fax: 617-264-6848, zobermeyer@ partners.org. Alternate: Alissa Clarke, AB, Department of Emergency Medicine, Brigham \& Women's Hospital, Neville House, 75 Francis St, Boston, MA 02115, aclarke1@partners.org.

Conflict of Interest: The authors have no relevant conflicts of interest to report.

Sponsor's Role: The funders had no role in the design and conduct of the study, in the collection, analysis, and interpretation of the data, or in the preparation, review, or approval of the manuscript.

Author Contributions: Study concept and design: ZO, DMC. Analysis and interpretation of data: ZO, MM. Literature review: ZO, AC. Drafting and revision of manuscript: ZO, AC, MM, JS, DMC. Obtained funding: ZO. Study supervision: ZO, DMC. 
during inpatient stays originating in EDs; they accounted for 35.7\% (95\% CI 35.4\%-36.0\%) of all hospice stays $<1$ month compared to $13.9 \%$ (95\% CI 13.6\%-14.2\%) of stays $>1$ month.

Conclusion-Most Medicare beneficiaries with poor-prognosis cancer visited EDs at the end of life. Hospice enrollment was associated with lower ED utilization and admission rates vs nonhospice care. Many patients enrolled in hospice during inpatient stays that followed ED visits, a phenomenon linked to shorter hospice stays. These findings must be interpreted carefully given potential unmeasured confounders in matching.

\section{Keywords}

hospice; emergency medicine; end-of-life care; Medicare

\section{INTRODUCTION}

Fifty-one percent of elderly patients visit the Emergency Department (ED) in the last month of life. ${ }^{1}$ The decisions made for these patients in the ED—about mechanical ventilation, invasive procedures, intensive care admission, and other high-intensity interventions ${ }^{2-4}$ have immediate and downstream implications for patients' care and quality of life, as well as for health care costs. ${ }^{2,5,6} \mathrm{As}$ a result, there is growing interest in promoting palliative and hospice care in the emergency setting. 7,8

While it has been shown that hospice enrollment in elderly Medicare patients is associated with fewer ED visits in the last month of life, ${ }^{1}$ significant questions remain regarding how much of this effect can be attributed to hospice: patients who choose hospice may simply be less likely to seek emergency care irrespective of their hospice enrollment, and there is little evidence on the trajectories of ED utilization before and after enrollment. In addition, the pattern of hospital admissions resulting from ED visits among hospice patients compared to non-hospice patients is unknown. As a result, the impact of hospice on ED utilization, and, more broadly, the relationship between emergency care and hospice, remain poorly understood.

We studied patterns of ED utilization and ED admissions before and after hospice enrollment in a large cohort of Medicare beneficiaries with poor-prognosis cancer. We compared those enrolled in the Medicare hospice benefit to matched controls receiving nonhospice care over the last year of life, and investigated the association of hospice care with ED utilization and ED admission rates over similar periods before death. We hoped to provide a more complete picture of the relationship between hospice and ED utilization and inform current debates about the role of palliative care in the ED.

\section{METHODS}

\section{Study Design and Participants}

We conducted a matched case-control study of all hospice-eligible, fee-for-service Medicare beneficiaries with poor-prognosis cancer who died in 2011. We adapted a palliative care screening instrument from a large cancer referral center to generate a list of International Classification of Disease (ICD) codes corresponding to poor-prognosis malignancies, which 
we define as those with a high risk of death within six months, including poor-prognosis primary cancers (e.g., lung, pancreatic, brain), any metastatic malignancy, and any hematologic malignancy specifically designated as unremitted or relapsed. ${ }^{9}$ The diagnoses used in the algorithm were developed by clinicians treating a wide range of cancer patients to identify those with poor prognoses and lack of options for curative treatments. Beneficiaries with any one of these ICD codes in the Medicare 100\% inpatient, outpatient, and hospice files from 2008 to 2011 were considered eligible for hospice, which is available to those with terminal illness and expected survival of less than 6 months, and were included, correcting for use of outpatient "rule-out" codes. ${ }^{10}$ Both hospice beneficiaries and non-hospice controls were thus eligible to enroll in hospice. Hospice enrollees were identified as those with at least 1 hospice claim submitted after the first cancer diagnosis.

To verify that this method successfully captured patients at high-risk of mortality, we calculated one-year mortality in a prior year of Medicare data. The mortality rate of this cohort, $31 \%$ in the year after first diagnosis, was similar to and only slightly lower than mortality rates in previous research on poor-prognosis cancer. ${ }^{11}$

Our method of matching hospice enrollees with non-hospice controls is described in more detail elsewhere. ${ }^{12}$ Briefly, we first matched beneficiaries according to individual-level characteristics, performing one-to-one exact matching of hospice beneficiaries to nonhospice patients on hospital referral region, age, sex, and time from first poor-prognosis cancer diagnosis to death (in months). This last variable was used as a proxy for disease course and severity. Because cancer patients tend to experience a decline in health at least 3 months before death, ${ }^{13}$ we assumed that both non-hospice and hospice beneficiaries included would likely have had sufficient time to consider and enroll in hospice. With their diagnoses of poor-prognosis cancer, all patients in both groups were eligible.

Using a strategy of progressively coarsening exact matching (CEM),${ }^{14}$ beneficiaries were matched by the finest strata of each variable (ZIP code, year of birth, sex, months from diagnosis to death). Remaining unmatched cases were then matched iteratively in increasingly broad categories, up to a maximum of 5-year age intervals, 4-month illness duration intervals, and hospital referral region (HRR). We had initially attempted propensity score matching (PSM), but this generated significant imbalances on important covariates (eTable 1); for example, only $0.8 \%$ of matched pairs resided in the same HRR. Thus, we present CEM results here, with PSM sensitivity analysis in the supplemental material; because of the computationally-intensive nature of PSM, we used a nationally-representative $20 \%$ sample of Medicare beneficiaries rather than the $100 \%$ sample used for CEM.

\section{Exposure Period}

For each pair of matched hospice and non-hospice (control) patients, we defined an exposure period leading up to death. The exposure period was defined as the number of days the hospice enrollee claimed the benefit until death. For each matched pair, this exposure period was applied to the non-hospice control, counting backwards from the control patient's death. While the chronological time of death differed between the hospice beneficiaries and non-hospice patients, the exposure time before death was equivalent. For 
example, if a beneficiary claimed hospice for 20 days prior to his or her death, the exposure period for the matched control would also be 20 days prior to the control patient's death.

\section{Outcomes}

We calculated the percent of beneficiaries visiting the ED in the last six months of life; sixmonth survival is the eligibility criterion for hospice enrollment in beneficiaries with terminal illness. We compared ED utilization and ED admission rates between hospice beneficiaries and matched non-hospice patients at baseline (defined as 1 year prior to the first week of hospice); the week prior to hospice enrollment; and the week prior to death, taking into account the varying duration of the exposure period (in weeks) between hospice enrollment and death.

\section{Statistical Analysis}

Balance between case and control groups was confirmed for all variables used for matching by comparing group means or medians. Utilization of health services and medical comorbidity at baseline was measured using the Gagne comorbidity score, a composite mortality scale based on Elixhauser and Charlson indices. ${ }^{15}$ Variables describing health services utilization and comorbidity at baseline were not used for matching, because the exposure periods for controls were defined retrospectively based on that of their matched hospice-enrolled pair. ED utilization was calculated per 1000 beneficiaries per day, and ED admission rate was calculated as the number of beneficiaries with an ED visit leading to an inpatient hospitalization as a percent of all beneficiaries visiting the ED. We compared both rates in case and control groups using risk ratios. Finally, we hypothesized that inpatient admission from an ED visit triggered hospice enrollment for some beneficiaries. We calculated the percent of hospice beneficiaries who enrolled during inpatient stays following admission from the ED, and explored the correlation between this phenomenon and length of hospice stay, which has been linked to quality of care. ${ }^{16-19}$

\section{RESULTS}

\section{Study Population and Characteristics}

Figure 1 shows the study population and the matching process. A total of 1,572,326

Medicare fee-for-service beneficiaries who died in 2011 were considered for inclusion. Of these, 420,503 had a prior diagnosis of poor-prognosis malignancy, and were thus eligible for hospice. We excluded 1,322 (0.31\%) beneficiaries who had missing or non-US ZIP codes, 2,462 (0.59\%) who had previously enrolled in hospice, and 12 with invalid dates (e.g., hospice or diagnosis dates following death date). In the remaining group, 254,729 patients claimed hospice for a median duration of 15 days (interquartile range [IQR] 5-47), compared to 161,978 patients who did not. The final study cohort consisted of 136,416 matched pairs (84.2\% of the non-hospice group).

Table 1 shows baseline characteristics of the study cohort. There were no statistically significant differences between hospice beneficiaries and non-hospice patients for any variables used for matching. Median comorbidity, inpatient admission, and ED visit rates were similar from 2006 to the beginning of the exposure period for both groups. Baseline 
differences in home health days, clinic visits, and daily expenses in the year prior to hospice were statistically significant: hospice beneficiaries had more days of home health assistance (median: 6 vs 4, difference: 2 [95\% CI: 1.7-2.3]) and more clinic visits (median: 1 vs 0 , difference: 1 [95\% CI: $0.87-1.1]$ ), but lower mean daily expenses in the year prior to hospice ( $\$ 132.3$ vs $\$ 141.5$, difference: $\$ 8.5$ [95\% CI: 7.5-9.5]). eTable 2 compares the study population to the overall population of all Medicare poor-prognosis cancer deaths in 2011, showing no differences between the included study population and the broader population in terms of age or sex. However, the median time from diagnosis to death was 6 weeks shorter for the study population than for the overall population: the distribution of illness duration was right-skewed (i.e., more patients with shorter survival and a long tail of patients with survival times above the median), making it more difficult to match beneficiaries with above-median illness duration and resulting in a likewise right-skewed matched cohort.

\section{ED Utilization}

For the entire study population, $81.3 \%$ (95\% CI: 81.1\%-81.4\%) had an ED visit in the last 6 months of life. For hospice beneficiaries, the median hospice stay was 14 days; under 3.7\% of stays exceeded 6 months. Figure 2 compares the number of ED visits per 1,000 beneficiaries per day for non-hospice patients and hospice beneficiaries, from 1 year before exposure period until death. We separated beneficiaries into groups based on the length of exposure period (i.e., time from hospice enrollment to death, or equivalent period for nonhospice controls). Because it was not feasible to show all 109 groups, and because aggregating different exposure lengths obscured time trends, we show representative groups with exposure periods of 1,2, and 4 weeks, which together make up $56.4 \%$ of the entire cohort, and every 4 weeks from 8 to 30 weeks, which make up $6.1 \%$ of the cohort. For each exposure period, the number of ED visits after enrollment was higher for the non-hospice patients than for hospice beneficiaries. Overall, $86.6 \%$ (CI: 86.4\%-86.8\%) of non-hospice patients were seen in the ED in the last 6 months of life, compared to $75.9 \%$ (CI: 75.7\%$76.2 \%$ ) in the hospice group (absolute difference: $10.7 \%$, CI: 10.4\%-11.0\%).

These trends are summarized in Table 2, which shows the number of ED visits per 1000 beneficiaries per day at baseline (from 2006 until 1 week before exposure period), 1 week before exposure, and 1 week before death. The ED visit rate was similar for hospice beneficiaries and non-hospice patients in the baseline period (rate ratio: 0.99, 95\% CI: 0.98 0.99). In the week before exposure period start, non-hospice patients were approximately half as likely as hospice beneficiaries to visit the ED (46.1 vs 88.1 visits per 1000 beneficiary-days, rate ratio: $0.56,95 \%$ CI: $0.56-0.57)$. In the final week of life, however, non-hospice patients were almost 10 times more likely than hospice beneficiaries to visit the ED (69.6 vs 7.6, rate ratio: 9.7, 95\% CI: 9.3-10.0). Non-hospice patients also visited the ED 10 times more often in the week prior to death than they did at baseline (5.4 at baseline vs 69.6 at 1 week before death), while hospice enrollees' ED visits increased only slightly (5.2 at baseline vs 7.6 at 1 week before death).

\section{ED Admission Rates}

There was no difference in ED admission rates between non-hospice patients and hospice beneficiaries at baseline (rate ratio: 1.0, 95\% CI 1.0-1.0) or in the week prior to exposure 
period start (rate ratio: $0.99,95 \%$ CI $0.99-1.0)$. In the final week of life, non-hospice patients in the ED were 51\% more likely to be admitted than hospice beneficiaries (rate ratio: $1.51,95 \%$ CI: $1.45-1.57)$. Non-hospice patients were admitted at higher rates in the week prior to death (rate: $0.63,95 \%$ CI $0.63-0.64$ ) than they were at baseline (rate: 0.55 , $95 \%$ CI $0.55-0.55)$, while the opposite was true for hospice beneficiaries ( 0.42 at 1 week before death vs 0.54 at baseline).

\section{Hospice Enrollment Following ED Admission}

Of all hospice beneficiaries, $28 \%$ enrolled during inpatient stays following admission from the ED. We explored the correlation between ED admission and time between hospice enrollment and death, and found that beneficiaries who enrolled in hospice after being admitted through the ED were more likely to have short hospice stays than those who did not enroll as inpatients. For beneficiaries with less than 1 month of hospice care, 35.7\% (95\% CI 35.4\%-36.0\%) started hospice as inpatients after ED admission, while this was only true for $13.9 \%$ (95\% CI 13.6\%-14.2\%) of those claiming hospice benefits for more than 1 month. This trend is summarized graphically in eFigure 1.

\section{Sensitivity Analysis}

PSM produced 100\% matching of the non-hospice group, for an included cohort of 69,854 beneficiaries. In this cohort, balance on important drivers of cost, most notably geography, was poor, with only $0.8 \%$ of matched pairs residing in the same HRR (eTable 1).

Nonetheless, general trends in ED visits and ED admissions were similar in PSM and CEM (eFigure 2), as were rates of ED visits and ED admissions (eTable 3).

\section{DISCUSSION}

While a vast majority of Medicare beneficiaries with poor-prognosis cancer visited EDs at the end of life, those enrolled in hospice had significantly fewer ED visits than matched nonhospice patients after hospice enrollment, despite generally similar patterns of ED utilization before enrollment. When hospice beneficiaries did visit the ED, they were also less likely to be hospitalized than non-hospice patients. We also identified a large minority of hospice beneficiaries who enrolled as inpatients following inpatient admission from the ED, a phenomenon linked to shorter hospice stays.

While the lower rate of ED visits among hospice patients had been identified in previous, smaller studies, ${ }^{1,20}$ an exact understanding of the nature of the correlation demanded a more careful examination of the temporal relationship between the two: because the hospice program requires beneficiaries to forgo curative care, it might be more attractive to patients with a baseline preference for less care. We found that ED utilization was similar between hospice and non-hospice patients at baseline, and observed a significant decrease in ED utilization immediately after hospice enrollment; there was no such pattern in non-hospice patients. Given the temporal pattern, this result was unlikely to be a reflection of baseline differences between hospice and non-hospice populations. The pattern could reflect changes in underlying health status that precipitate hospice enrollment, or a causal effect of choosing hospice. Such causality, however, cannot be determined with this retrospective data. We 
also found that inpatient admissions from the ED decreased for hospice beneficiaries after enrollment. Given studies suggesting that most patients prefer to stay out of the ED and hospitals in the days leading up to death, ${ }^{21}$ the reduction in ED use and admissions indicates that hospice is an important correlate of quality of care at the end of life.

Our findings also highlight important connections between ED utilization, hospital admission, and hospice enrollment, building on prior studies. ${ }^{22-25} \mathrm{We}$ found that nearly onethird of hospice beneficiaries enrolled as inpatients following ED admission, and that these beneficiaries had shorter hospice stays. This phenomenon likely reflects both the growing role of palliative and hospice interventions in the emergency setting and shortly afterwards, and also failures to address palliative care needs earlier in the outpatient setting, ${ }^{26}$ as demonstrated in previous work connecting late referrals, emergency utilization, and aggressive end-of-life care. ${ }^{16}$ Visits to the ED, and especially admissions from the ED, may represent an important "pause point" for patients with poor-prognosis cancers, and a window of opportunity for emergency care providers to engage with patients in end-of-life care conversations, which could be continued in more depth by oncologists, hospitalists, intensivists, and other providers in the hospital. However, given that these patients had shorter hospice stays, which are increasingly considered indicative of lower end-of-life quality of care, ${ }^{16-19}$ hospice enrollment in the emergency setting or shortly thereafter is unlikely to represent optimal care. Our results reinforce the need to increase discussion of hospice and palliative care at multiple points in the care of patients with advanced illnesses -including the ED and inpatient hospitalizations, but especially the routine outpatient setting.

There were limitations to this study. In matching, we made assumptions about the correlation between illness severity and illness length: first, that the two were in fact correlated; second, that hospice enrollment was not correlated with illness length. If hospice enrollment were correlated with shorter survival times, then hospice beneficiaries would have been healthier than they appeared from their illness length, a difference that could partially account for the decreased ED visit and ED admissions rates after enrollment. If, however, hospice enrollment were correlated with longer survival times, then hospice beneficiaries would have been sicker than they appeared from their illness length, a difference that would buffer the decrease in ED visit and admissions rates post-enrollment. Since there is some evidence that hospice care may prolong life, ${ }^{27}$ the latter seems more likely; our results may thus not capture the full reduction in ED visits and admissions among hospice beneficiaries after enrollment. The group of hospice beneficiaries had higher comorbidity scores, indicating that they may have been sicker than their non-hospice controls. However, if we are correct that illness length was correlated with disease severity, the matching process would have corrected for any such discrepancies.

It is additionally important to point out that while matching according to age, sex, geography, and illness severity captured relevant similarities between two matched patients, we were unable to account for all potential variables and thus cannot say with certainty that the two groups were fully comparable. It is reassuring that, despite matching on only four variables, we were able to achieve balance on a wide range of other measured variables, including median comorbidity, inpatient admissions, and ED visits, increasing our 
confidence in the matching procedure. However, it is impossible to assess balance on unmeasured covariates, and our results must be interpreted in this light.

There are also potential limitations to generalizability. In order to create a matched cohort balanced on illness duration, demographics, and geography, we excluded a subset of unmatched beneficiaries, potentially biasing results, though additional sensitivity analysis with PSM matched all beneficiaries and produced largely similar results. Other limitations included the restricted analysis to all fee-for-service Medicare beneficiaries with cancer and incomplete data on skilled nursing facility expenses.

Finally, we identified patients with poor-prognosis cancer and high risk of mortality through ICD codes in their Medicare claims files. Such claims-based diagnoses can be inaccurate. These codes may also include cancers that range in severity, though we did specify ICD codes to exclude more indolent cancers with better prognoses (e.g., restricting to hematologic malignancies specifically designated as relapsed or not in remission).

Additionally, there is, to the authors' knowledge, no data on the specificity and sensitivity of these codes in predicting survival. However, we verified that this method did indeed identify high-mortality subgroups by calculating one-year mortality in a year of prior claims data, finding $31 \%$ mortality. The high mortality of this group suggested that using ICD codes was an effective method to identify patients with a high risk of near-term death. Lastly, all studies on 'decedents' encounter an important limitation in identifying patients at high risk of death, since patients' prognoses may not always be clear to themselves or their providers. We also assume that patients with such poor prognoses were knowledgeable about their eligibility for hospice, when patients may have had hospice presented to them as an option late in the course of their illness and then only after a hospitalization.

In conclusion, most Medicare beneficiaries with poor-prognosis cancer visited EDs at the end of life. Hospice enrollment was associated with decreases in emergency care intensity, including lower ED utilization and rates of inpatient admission compared to non-hospice care. Nearly one-third of hospice beneficiaries enrolled in hospice as inpatients following an ED admission, likely reflecting failures to address palliative care needs in the outpatient setting, and this phenomenon was linked to shorter hospice stays.

\section{Supplementary Material}

Refer to Web version on PubMed Central for supplementary material.

\section{Acknowledgments}

Funding sources: NIH (Common Fund/Office of the Director), DP5 OD012161 (PI: Obermeyer)

\begin{tabular}{|c|c|c|c|c|c|c|c|c|c|c|}
\hline \multirow[t]{2}{*}{ Elements of Financial/Personal Conflicts } & \multicolumn{2}{|c|}{$\begin{array}{l}\text { *Author } 1 \\
\text { ZO }\end{array}$} & \multicolumn{2}{|c|}{$\begin{array}{l}\text { Author } 2 \\
\text { ACC }\end{array}$} & \multicolumn{2}{|c|}{$\begin{array}{l}\text { Author } 3 \\
\text { MM }\end{array}$} & \multicolumn{2}{|c|}{$\begin{array}{l}\text { Author } 4 \\
\text { JDS }\end{array}$} & \multicolumn{2}{|c|}{$\begin{array}{l}\text { Author } 5 \\
\text { DMC }\end{array}$} \\
\hline & Yes & No & Yes & No & Yes & No & Yes & No & Yes & No \\
\hline Employment or Affiliation & &  & & $\bar{x}$ & & $\mathrm{x}$ & & $\mathrm{x}$ & & $\mathrm{x}$ \\
\hline Grants/Funds & & $\mathrm{x}$ & & $\mathrm{x}$ & & $\mathrm{x}$ & & $\mathrm{x}$ & & $\mathrm{x}$ \\
\hline
\end{tabular}




\begin{tabular}{|l|l|l|l|l|l|l|l|l|l|l|l|}
\hline Elements of Financial/Personal Conflicts & \multicolumn{2}{l}{$\begin{array}{l}\text { *Author 1 } \\
\text { ZO }\end{array}$} & \multicolumn{2}{l|l|l|l|l|l|}{$\begin{array}{l}\text { Author 2 } \\
\text { ACC }\end{array}$} & \multicolumn{2}{l|}{$\begin{array}{l}\text { Author 3 } \\
\text { MM }\end{array}$} & \multicolumn{2}{l|}{$\begin{array}{l}\text { Author 4 } \\
\text { DMC }\end{array}$} \\
\hline & Yes & No & Yes & No & Yes & No & Yes & No & Yes & No \\
\hline Honoraria & & $\mathrm{x}$ & & $\mathrm{x}$ & & $\mathrm{x}$ & & $\mathrm{x}$ & & $\mathrm{x}$ \\
\hline Speaker Forum & & $\mathrm{x}$ & & $\mathrm{x}$ & & $\mathrm{x}$ & & $\mathrm{x}$ & & $\mathrm{x}$ \\
\hline Consultant & & $\mathrm{x}$ & & $\mathrm{x}$ & & $\mathrm{x}$ & & $\mathrm{x}$ & & $\mathrm{x}$ \\
\hline Stocks & & $\mathrm{x}$ & & $\mathrm{x}$ & & $\mathrm{x}$ & & $\mathrm{x}$ & & $\mathrm{x}$ \\
\hline Royalties & & $\mathrm{x}$ & & $\mathrm{x}$ & & $\mathrm{x}$ & & $\mathrm{x}$ & & $\mathrm{x}$ \\
\hline Expert Testimony & & $\mathrm{x}$ & & $\mathrm{x}$ & & $\mathrm{x}$ & & $\mathrm{x}$ & & $\mathrm{x}$ \\
\hline Board Member & & $\mathrm{x}$ & & $\mathrm{x}$ & & $\mathrm{x}$ & & $\mathrm{x}$ & & $\mathrm{x}$ \\
\hline Patents & & $\mathrm{x}$ & & $\mathrm{x}$ & & $\mathrm{x}$ & & $\mathrm{x}$ & & $\mathrm{x}$ \\
\hline Personal Relationship & & $\mathrm{x}$ & & $\mathrm{x}$ & & $\mathrm{x}$ & & $\mathrm{x}$ & & $\mathrm{x}$ \\
\hline
\end{tabular}

The authors gratefully acknowledge the help of the Research Data Assistance Center Help Desk at the University of Minnesota. Stacey Tobin, $\mathrm{PhD}$ (The Tobin Touch, LLC) contributed to this manuscript and was compensated for editorial assistance.

\section{References}

1. Smith AK, McCarthy E, Weber E, et al. Half of older Americans seen in emergency department in last month of life; most admitted to hospital, and many die there. Health Aff (Millwood). 2012; 31:1277-1285. [PubMed: 22665840]

2. Barnato AE, McClellan MB, Kagay CR, et al. Trends in inpatient treatment intensity among Medicare beneficiaries at the end of life. Health Serv Res. 2004; 39:363-375. [PubMed: 15032959]

3. Wright AA, Zhang B, Ray A, et al. Associations between end-of-life discussions, patient mental health, medical care near death, and caregiver bereavement adjustment. JAMA. 2008; 300:16651673. [PubMed: 18840840]

4. Schuur JD, Hsia RY, Burstin H, et al. Quality measurement in the emergency department: Past and future. Health Aff (Millwood). 2013; 32:2129-2138. [PubMed: 24301396]

5. Riley GF, Lubitz JD. Long-term trends in Medicare payments in the last year of life. Health Serv Res. 2010; 45:565-576. [PubMed: 20148984]

6. Kelley AS, Deb P, Du Q, et al. Hospice enrollment saves money for Medicare and improves care quality across a number of different lengths-of-stay. Health Aff (Millwood). 2013; 32:552-561. [PubMed: 23459735]

7. Choosing Wisely. [Accessed March 10, 2014] Five Things Physicians and Patients Should Question. 2013. (online) Available at: http://www.choosingwisely.org/doctor-patient-lists/americancollege-of-emergency-physicians/

8. Beemath A, Zalenski RJ. Palliative emergency medicine: resuscitating comfort care? Ann Emerg Med. 2009; 54:103-105. [PubMed: 19346031]

9. Glare, P.; Christakis, NA. Prognosis in Advanced Cancer. New York, NY: Oxford University Press; 2008.

10. Wennberg JE, Staiger DO, Sharp SM, et al. Observational intensity bias associated with illness adjustment: Cross sectional analysis of insurance claims. BMJ. 2013; 346:f549. [PubMed: 23430282]

11. Sima CS, Panageas KS, Schrag D. Cancer screening among patients with advanced cancer. JAMA. 2010; 304:1584-1591. [PubMed: 20940384]

12. Obermeyer Z, Makar M, Abujaber S, et al. Association between the Medicare hospice benefit and health care utilization and costs for patients with poor-prognosis cancer. JAMA. 2014; 312:18881896. [PubMed: 25387186] 
13. Gill TM, Gahbauer EA, Han L, et al. Trajectories of disability in the last year of life. N Engl J Med. 2010; 362:1173-1180. [PubMed: 20357280]

14. Iacus SM, King G, Porro G. Multivariate matching methods that are monotonic imbalance bounding. J Am Stat Assoc. 2011; 106:345-361.

15. Gagne JJ, Glynn RJ, Avorn J, et al. A combined comorbidity score predicted mortality in elderly patients better than existing scores. J Clin Epidemiol. 2011; 64:749-759. [PubMed: 21208778]

16. Hui D, Kim SH, Roquemore J, et al. Impact of timing and setting of palliative care referral on quality of end-of-life care in cancer patients. Cancer. 2014; 120:1743-1749. [PubMed: 24967463]

17. Teno JM, Gozalo PL, Bynum JW, et al. Change in end-of-life care for Medicare beneficiaries: Site of death, place of care, and health care transitions in 2000, 2005, and 2009. JAMA. 2013; 309:470-477. [PubMed: 23385273]

18. O'Connor NR, Hu R, Harris PS, et al. Hospice admissions for cancer in the final days of life: Independent predictors and implications for quality measures. J Clin Oncol. 2014; 32:3184-3189. [PubMed: 25154824]

19. Teno JM, Shu JE, Casarett D, et al. Timing of referral to hospice and quality of care: Length of stay and bereaved family members' perceptions of the timing of hospice referral. J Pain Symptom Manage. 2007; 34:120-125. [PubMed: 17583469]

20. Bergman J, Saigal CS, Lorenz KA, et al. Hospice use and high-intensity care in men dying of prostate cancer. Arch Intern Med. 2011; 171:204-210. [PubMed: 20937914]

21. Higginson IJ, Sen-Gupta GJ. Place of care in advanced cancer: A qualitative systematic literature review of patient preferences. J Palliat Med. 2000; 3:287-300. [PubMed: 15859670]

22. Smith AK, Fisher J, Schonberg MA, et al. Am I doing the right thing? Provider perspectives on improving palliative care in the emergency department. Ann Emerg Med. 2009; 54:86-93. 93 e81. [PubMed: 18930337]

23. Grudzen CR, Richardson LD, Hopper SS, et al. Does palliative care have a future in the emergency department? Discussions with attending emergency physicians. J Pain Symptom Manage. 2012; 43:1-9. [PubMed: 21802899]

24. Grudzen CR, Richardson LD, Major-Monfried H, et al. Hospital administrators' views on barriers and opportunities to delivering palliative care in the emergency department. Ann Emerg Med. 2013; 61:654-660. [PubMed: 22771203]

25. Quest T, Herr S, Lamba S, et al. Demonstrations of clinical initiatives to improve palliative care in the emergency department: A report from the IPAL-EM Initiative. Ann Emerg Med. 2013; 61:661-667. [PubMed: 23548402]

26. Grudzen CR, Stone SC, Morrison RS. The palliative care model for emergency department patients with advanced illness. J Palliat Med. 2011; 14:945-950. [PubMed: 21767164]

27. Temel JS, Greer JA, Muzikansky A, et al. Early palliative care for patients with metastatic nonsmall-cell lung cancer. N Engl J Med. 2010; 363:733-742. [PubMed: 20818875]

$J$ Am Geriatr Soc. Author manuscript; available in PMC 2017 February 01. 




Figure 1. Study population

(A) Generation of the matched cohort. We first identified all Medicare beneficiaries who died in 2011, then selected those diagnosed with poor-prognosis cancer. Beneficiaries with prior hospice enrollment suggesting a preceding terminal illness, as well as those with missing geographic or date of death information were excluded. The remaining set was divided by hospice enrollment and then matched, with $84.2 \%$ of the non-hospice group paired. 


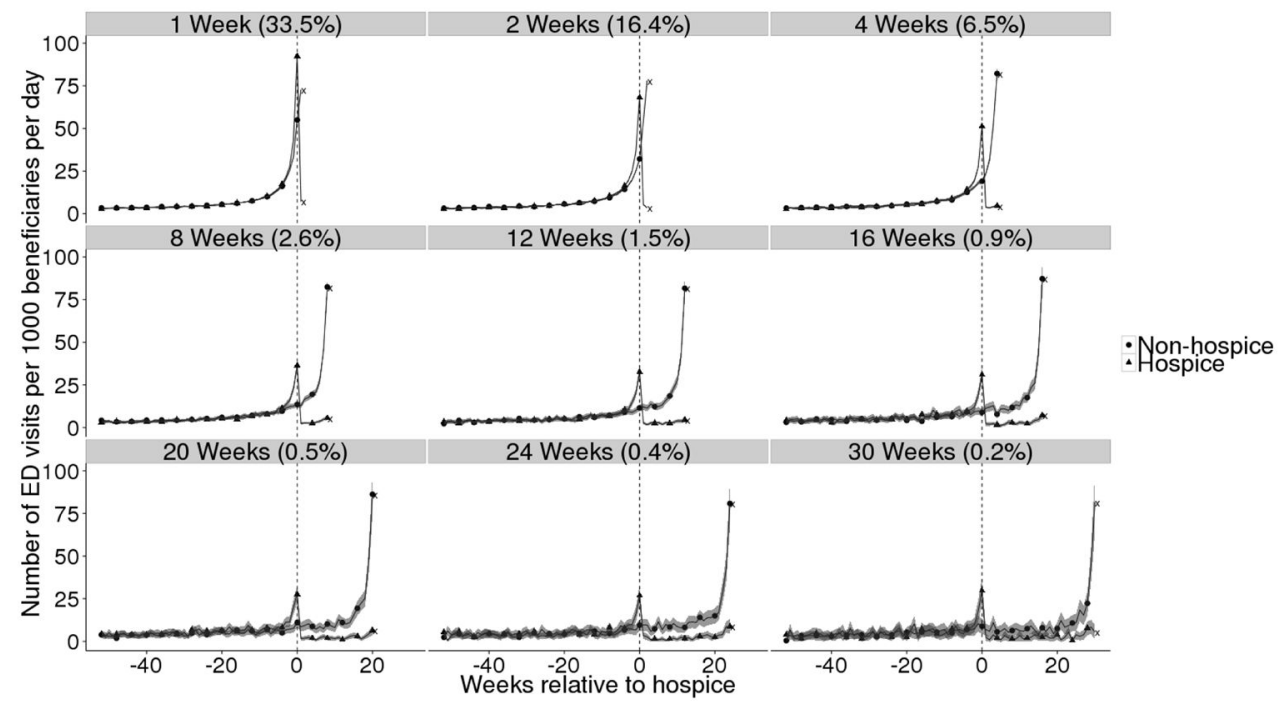

Figure 2. ED visit rate for non-hospice patients and hospice beneficiaries

The mean daily number of ED visits per 1000 beneficiaries for non-hospice patients and hospice beneficiaries, from 1 year before the start of the exposure period (week 0) until death. We separated beneficiaries into groups based on the length of exposure period (i.e., the length of hospice or non-hospice care before death). Because we could not show all 109 groups, and aggregating groups would obscure time trends, we show representative groups with exposure periods of 1,2 , and 4 weeks, which together make up $56.4 \%$ of the entire cohort, and then at 4 week intervals from 8 to 30 weeks, which make up $6.1 \%$ of the cohort. Crosses mark the week of death for each group of beneficiaries. The vertical dash line at week 0 marks hospice enrollment and the start of the exposure period. The shaded area around the lines indicates the $95 \%$ confidence interval for the mean daily visits. 


\section{Table 1}

\section{Study Population Characteristics}

Table 1 shows baseline characteristics of the study cohort.

\begin{tabular}{|c|c|c|c|c|}
\hline Variable & & Non-hospice $(\mathrm{N}=\mathbf{1 3 6}, \mathbf{4 1 6})$ & Hospice (N=136,416) & Difference $^{a}$ \\
\hline \multirow{17}{*}{ Baseline period $^{a}$} & Characteristics used for matching & & & \\
\hline & Age (years) ${ }^{b}$ & $77.5(77.5,77.7)$ & $77.5(77.5,77.6)$ & $-0.01(-0.09,0.06)$ \\
\hline & Male sex $(\%)^{b}$ & $50.2(50.0,50.4)$ & $50.2(50.0,50.4)$ & $0(0,0)$ \\
\hline & Median distance between pairs (miles) ${ }^{c}$ & \multicolumn{2}{|c|}{$18.9(7.5,45.0)$} & - \\
\hline & Time from diagnosis to death (days) ${ }^{c}$ & $213(45,646)$ & $212(49,645)$ & $-1(-5.2,3.2)$ \\
\hline & Illness and hospice course & & & \\
\hline & From diagnosis to exposure (days) ${ }^{c}$ & $168(23,601)$ & $168(23,600)$ & $0(-4,4)$ \\
\hline & From exposure start to death (days) ${ }^{c}$ & $14(4,42)$ & $14(4,42)$ & $0(0,0)$ \\
\hline & Comorbidity prior to exposure ${ }^{d}$ & & & \\
\hline & From 2006 to cancer diagnosis ${ }^{c}$ & $6(3,9)$ & $6(3,8)$ & $0(0,0)$ \\
\hline & From 2006 to start of exposure period ${ }^{c}$ & $8(5,10)$ & $8(6,11)$ & $0(0,0)$ \\
\hline & $\begin{array}{l}\text { Healthcare utilization from } 2006 \text { to start of } \\
\text { exposure period }\end{array}$ & & & \\
\hline & Inpatient admissions ${ }^{c}$ & $3(1,5)$ & $3(1,5)$ & $0(0,0)$ \\
\hline & Emergency visits ${ }^{c}$ & $4(2,7)$ & $4(2,7)$ & $0(0,0)$ \\
\hline & Home health days ${ }^{c}$ & $4(0,27)$ & $6(0,27)$ & $2(1.7,2.3)$ \\
\hline & Clinic visits ${ }^{c}$ & $0(0,7)$ & $1(0,7)$ & $1(0.87,1.1)$ \\
\hline & Daily expenses in the year prior to hospice & $141.5(140.6,142.3)$ & $132.3(132.2,133.7)$ & $8.5(7.5,9.5)$ \\
\hline
\end{tabular}

${ }^{a}$ The baseline period is defined as the week one year before the first week of the exposure period. The last column shows differences between groups, calculated as described below.

${ }^{b}$ For normally-distributed variables, means are reported, with $95 \%$ confidence intervals in parentheses. Difference and $95 \%$ confidence interval are calculated by $\mathrm{t}$-test.

${ }^{c}$ For non-normally-distributed variables, medians are reported, with 25 th, 75 th percentiles in parentheses. Difference and $95 \%$ confidence interval are calculated by quantile regression.

${ }^{d}$ Gagne comorbidity score, measured on a composite scale synthesizing Elixhauser and Charlson indices. Scale ranges from -2 to 26. 
Table 2

ED Visit Rate and ED Admission Rate for Non-hospice Patients and Hospice Beneficiaries

Table 2 shows the number of ED visits per 1000 beneficiaries per day at baseline (from 2006 until 1 week before exposure period), 1 week before exposure, and 1 week before death.

\begin{tabular}{|llll|}
\hline & Non-hospice & Hospice & Ratio $^{a}$ \\
Variable & & & \\
Baseline & & & \\
$\quad$ ED visits per 1000 beneficiaries per day & $5.4(5.3,5.4)$ & $5.2(5.2,5.2)$ & $0.99(0.98,0.99)$ \\
ED Admission Rate & $0.55(0.55,0.55)$ & $0.54(0.54,0.55)$ & $1.0(1.0,1.0)$ \\
Week before exposure start & & & \\
ED visits per 1000 beneficiaries per day & $46.1(45.4,46.9)$ & $88.1(87.1,89.1)$ & $0.56(0.56,0.57)$ \\
ED Admission Rate & $0.77(0.77,0.78)$ & $0.78(0.78,0.78)$ & $0.99(0.99,1.0)$ \\
Week before death & & & \\
ED visits per 1000 beneficiaries per day & $69.6(68.3,70.9)$ & $7.6(7.3,8.0)$ & $9.7(9.3,10.0)$ \\
ED Admission Rate & $0.63(0.63,0.64)$ & $0.42(0.41,0.44)$ & $1.51(1.45,1.57)$ \\
\hline
\end{tabular}

${ }^{a}$ Ratio of hospice to non-hospice percentage, calculated as proportion of non-hospice patients over hospice beneficiaries, with $95 \%$ confidence interval in parentheses, calculated as a relative risk.

$b_{2006}$ to one week before the beginning of the exposure period. 